\title{
Identifying cooperative transcription factors in yeast using multiple data sources
}

\author{
Fu-Jou Lai', Mei-Huei Jhu², Chia-Chun Chiu², Yueh-Min Huang ${ }^{1}$, Wei-Sheng $\mathrm{Wu}^{2 *}$ \\ From The 25th International Conference on Genome Informatics (GIW/ISCB-Asia) \\ Tokyo, Japan. 15-17 December 2014
}

\begin{abstract}
Background: Transcriptional regulation of gene expression is usually accomplished by multiple interactive transcription factors (TFs). Therefore, it is crucial to understand the precise cooperative interactions among TFs. Various kinds of experimental data including ChIP-chip, TF binding site (TFBS), gene expression, TF knockout and protein-protein interaction data have been used to identify cooperative TF pairs in existing methods. The nucleosome occupancy data is not yet used for this research topic despite that several researches have revealed the association between nucleosomes and TFBSs.
\end{abstract}

Results: In this study, we developed a novel method to infer the cooperativity between two TFs by integrating the TF-gene documented regulation, TFBS and nucleosome occupancy data. TF-gene documented regulation and TFBS data were used to determine the target genes of a TF, and the genome-wide nucleosome occupancy data was used to assess the nucleosome occupancy on TFBSs. Our method identifies cooperative TF pairs based on two biologically plausible assumptions. If two TFs cooperate, then (i) they should have a significantly higher number of common target genes than random expectation and (ii) their binding sites (in the promoters of their common target genes) should tend to be co-depleted of nucleosomes in order to make these binding sites simultaneously accessible to TF binding. Each TF pair is given a cooperativity score by our method. The higher the score is, the more likely a TF pair has cooperativity. Finally, a list of 27 cooperative TF pairs has been predicted by our method. Among these 27 TF pairs, 19 pairs are also predicted by existing methods. The other 8 pairs are novel cooperative TF pairs predicted by our method. The biological relevance of these 8 novel cooperative TF pairs is justified by the existence of protein-protein interactions and co-annotation in the same MIPS functional categories. Moreover, we adopted three performance indices to compare our predictions with 11 existing methods' predictions. We show that our method performs better than these 11 existing methods in identifying cooperative TF pairs in yeast. Finally, the cooperative TF network constructed from the 27 predicted cooperative TF pairs shows that our method has the power to find cooperative TF pairs of different biological processes.

Conclusion: Our method is effective in identifying cooperative TF pairs in yeast. Many of our predictions are validated by the literature, and our method outperforms 11 existing methods. We believe that our study will help biologists to understand the mechanisms of transcriptional regulation in eukaryotic cells.

\section{Background}

Transcriptional regulation plays a crucial role in the regulation of gene expression. As well known, it is accomplished by the binding of transcription factors (TFs) to the TF

\footnotetext{
* Correspondence: wessonwu@mail.ncku.edu.tw

${ }^{2}$ Department of Electrical Engineering, National Cheng Kung University, No.1 University Road, 70101, Tainan, Taiwan

Full list of author information is available at the end of the article
}

binding sites (TFBSs) in the promoters of genes. In eukaryotic cells, transcriptional regulation is usually achieved by the cooperation between multiple TFs to regulate the expression of genes. Therefore, knowing the precise cooperative interactions among TFs is helpful for uncovering the mechanisms of transcriptional regulation.

With advances in high-throughput microarray technologies and diverse data sources, it is now possible to 
investigate the cooperative interactions among TFs. Many computational methods have been developed to identify cooperative TF pairs by using one or several kinds of experimental data. Some methods only used ChIP-chip data [1-3]. Several other methods integrated ChIP-chip and gene expression data [4-7]. Another two methods integrated ChIP-chip data with other data sources such as protein-protein interaction data [8] and TF knockout data [9]. On the contrary, Pilpel et al.'s method did not use ChIP-chip data but integrated TFBS and gene expression data [10]. Wang et al.'s and $\mathrm{Hu}$ et al.'s methods both integrated multiple data sources by using a Bayesian approach $[11,12]$.

ChIP-chip, gene expression, TFBS, TF knockout and protein-protein interaction data were used to investigate the cooperative interactions among TFs in the above mentioned methods. However, the nucleosome occupancy data was not used even though several researches have revealed the association between nucleosomes and TFBSs [13-16]. Because nucleosome occupancy has been demonstrated as an important strategy to regulate gene expression by affecting the accessibility of TFBSs to TFs, this biological knowledge motivates us to consider the effect of nucleosome occupancy on the cooperativity between TFs and adopt the nucleosome occupancy data for our research. Our method is developed based on the following two rationales. First, if two TFs cooperate, they should have a significantly higher number of common target genes than random expectation. Second, the TFBSs of these two cooperative TFs (in the promoters of their common target genes) should tend to be co-depleted of nucleosomes in order to make themselves simultaneously accessible to TF binding.

\section{Methods}

\section{Data sources}

We used three data sources in this study. First, the genome-wide nucleosome occupancy data of Saccharomyces cerevisiae was downloaded from Mavrich et al.'s study [17]. They established a genome-wide map of nucleosome locations and the map shows which region in the genome is occupied by nucleosomes. Second, the TF-gene documented regulation data was downloaded from YEASTRACT database [18], which deposited the documented regulation evidence (from the ChIP-chip and TF knockout experiments in the literature) between TFs and their target genes. Third, the TFBS data was downloaded from SwissRegulon database [19]. Each TFBS has its predicted genomic location and a posterior probability to indicate the confidence of this putative TFBS. In this study, a threshold 0.3 of the posterior probability was applied to select putative TFBSs. The total number of distinct TFs from the above two databases was 186, and therefore 17205 (186*185/2) TF pairs were considered in this study.

\section{The proposed method}

The proposed method is developed based on the following two biologically plausible assumptions. First, two cooperative TFs should share a significantly larger set of target genes than random expectation. This assumption has also been used in existing methods $[1,11,12,20]$. Second, the TFBSs of two cooperative TFs (in the promoters of their common target genes) should be co-depleted of nucleosomes to make themselves simultaneously accessible to TF binding. This assumption is biologically plausible since it has been shown that functionally cooperative TF pairs are associated with nucleosome-depleted promoters [21]. Therefore, given a TF pair, we calculate the significance of the overlap of their target genes and the significance of being co-depleted of nucleosomes on their TFBSs. Our method assigns a cooperativity score to each of the 17205 TF pairs. Finally, 27 TF pairs whose cooperativity scores larger than 120 are predicted as cooperative TF pairs. The flow chart of our method is shown in Figure 1 and described as follows.

\section{Step1-Define the target genes of each of the 186 TFs}

A TF's target genes are defined as those genes (i) that are known to be regulated by the TF from the TF-gene documented regulation evidence (retrieved from YEASTRACT database [18]) or (ii) whose promoters contain the binding sites of the TF (retrieved from SwissRegulon database [19]).

\section{Step2-Calculate the significance of the target gene overlap}

Given a TF pair, the significance of the overlap between the target genes of these two TFs is calculated using the Hyper-geometric test [22]:

$$
P_{\text {overlap }}=\sum_{i=m}^{\min \left(N_{1}, N_{2}\right)} \frac{\left(\begin{array}{c}
N_{1} \\
i
\end{array}\right)\left(\begin{array}{c}
N-N_{1} \\
N_{2}-i
\end{array}\right)}{\left(\begin{array}{c}
N \\
N_{2}
\end{array}\right)}
$$

where $N_{1}$ is the number of target genes of the first TF, $N_{2}$ is the number of target genes of the second TF, $N=$ 6576 is the number of total genes in the yeast genome, and $m$ is the number of common target genes. The smaller the $P_{\text {overlap }}$, the more significant the target gene overlap.

Step3-Calculate the significance of being co-depleted of nucleosomes on the TFBSs

From the common target genes of the given TF pair, we extract the genes which have both TFs' binding sites in their promoters, and denote it as set $A$. If multiple TFBSs of the same TF are found, only the most confident TFBS (with the highest posterior probability) is considered. Therefore, the promoter of each gene in set 


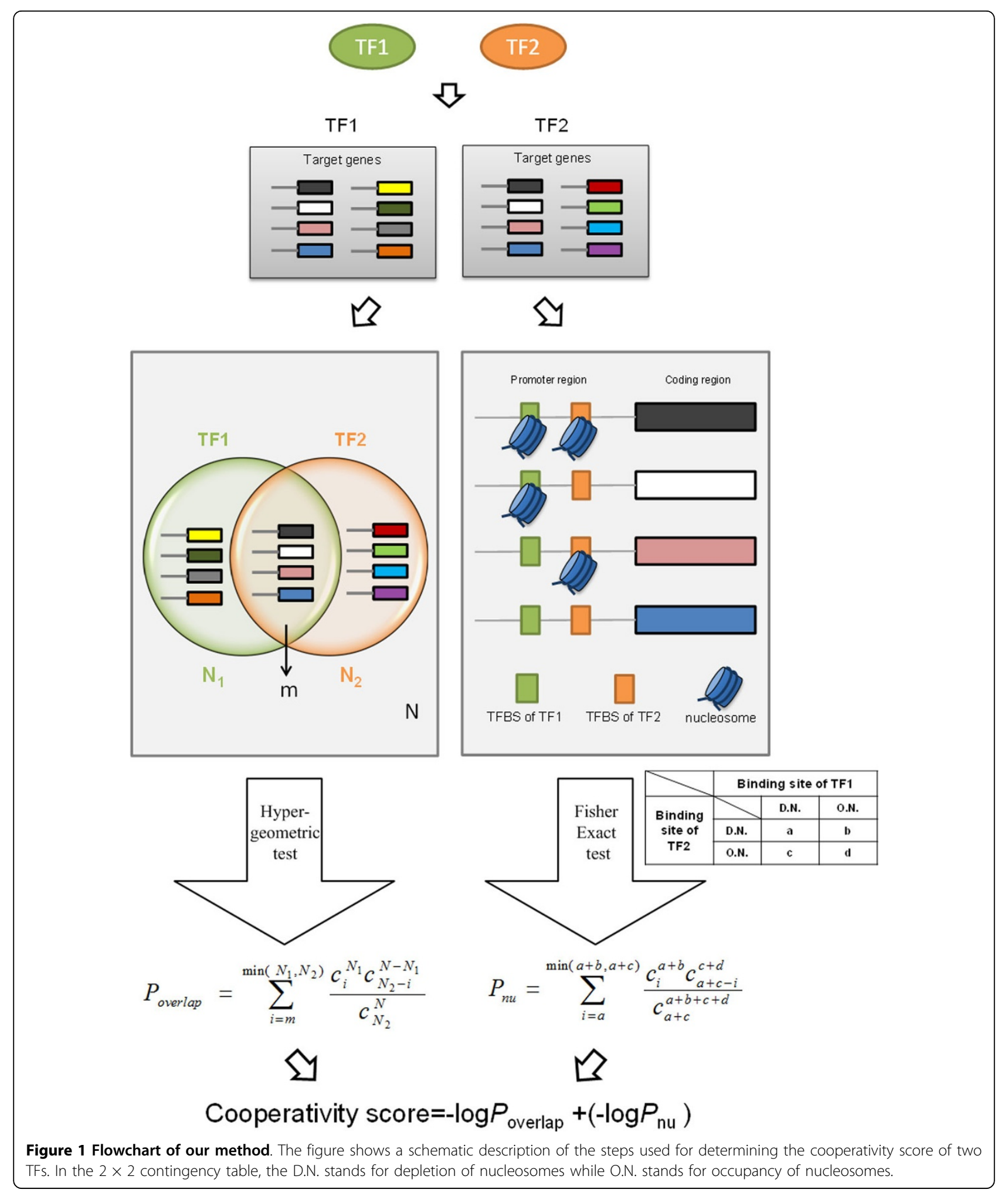

$A$ contains one TFBS of the first TF and one TFBS of the second TF.

For each gene in set $A$, the state of nucleosome occupancy of each of the two TFBSs can be specified to one of the two categories:

Occupied, if any position in the TFBS is occupied by a nucleosome. Depleted, if no position in the TFBS is occupied by a nucleosome. 
Then the state of nucleosome occupancy can be converted to a binary value and denoted as $S_{(T F B S j)}$ :

$$
S_{(T F B S j)}=\left\{\begin{array}{l}
1, \text { occupied } \\
0, \text { depleted }
\end{array}\right.
$$

where $j=1$ for the first TF and $j=2$ for the second TF. According to the values of $S_{(T F B S 1)}$ and $S_{(T F B S 2)}$, each gene in set $A$ can be assigned to a cell in a $2 \times 2$ contingency table (see Figure 1) and the number of genes in each of the four cells in the contingency table can be obtained. Then the Fisher exact test [23] is used to calculate the significance of TFBS1 and TFBS2 to be co-depleted of nucleosomes. The $p$-value is denoted as

$$
P_{n u}=\sum_{i=a}^{\min (a+b, a+c)} \frac{\left(\begin{array}{c}
a+b \\
i
\end{array}\right)\left(\begin{array}{c}
c+d \\
a+c-i
\end{array}\right)}{\left(\begin{array}{c}
a+b+c+d \\
a+c
\end{array}\right)}
$$

where $a$ is the number of genes whose promoters contain both TFBS1 and TFBS2 depleted of nuclesomes, $b$ is the number of genes whose promoters contain TFBS1 occupied by nucleosomes and TFBS2 depleted of nuclesomes, $c$ is the number of genes whose promoters contain TFBS1 depleted of nucleosomes and TFBS2 occupied by nuclesomes, and $d$ is the number of genes whose promoters contain both TFBS1 and TFBS2 occupied by nuclesomes. The smaller the $P_{n u}$, the more significant the TFBS1 and TFBS2 to be co-depleted of nucleosomes.

\section{Step4-Calculate the cooperativity score}

The cooperativity score is defined as $-\log P_{\text {overlap }}+\left(-\log P_{n u}\right)$. The higher the score is, the more likely a TF pair has cooperativity. There are two situations that can have a high score. One is a small $P_{\text {overlap}}$, i.e. the TF pair has a significant overlap between their target genes. The other situation is a small $P_{n u}$, i.e. the two TFs show a high tendency of being co-depleted of nucleosomes on their TFBSs to make them simultaneously accessible to TF binding.

\section{Results}

According to the cooperativity score, 17205 TF pairs can be sorted decreasingly, and then constitute a ranked prediction of cooperative TF pairs. Finally, 27 TF pairs whose cooperativity scores larger than 120 are predicted as cooperative TF pairs.

\section{Detailed investigation of the 27 predicted cooperative TF pairs}

In Table 1, we list these 27 predicted cooperative TF pairs. All of them have at least one of the following three lines of evidence: (i) being predicted by existing methods, (ii) the existence of protein-protein interactions, and (iii) the coannotation in the same MIPS functional categories. More precisely, $63 \%(17 / 27)$ of the pairs have all three lines of evidence, $26 \%(7 / 27)$ of the pairs have two lines of evidence, and $11 \%$ (3/27) of the pairs have only one line of evidence.

Note that among these 27 predicted cooperative TF pairs, 19 pairs are also predicted by existing methods. The other 8 pairs are novel cooperative TF pairs predicted by our method. The biological relevance of these 8 novel cooperative TF pairs is justified by the existence of protein-protein interactions and co-annotation in the same MIPS functional categories. More precisely, 75\% (6/8) of the novel pairs

(i.e. Pdr1-Pdr3, Dal80-Gln3, Pho4-Cbf1, Gal80-Gal4, Nrg1-Nrg2 and Hap5-Hap4) are highly biologically plausible since they have protein-protein interactions and are co-annotated in the same MIPS functional categories, and the other 25\% (2/8) of the novel pairs (i.e. Dal80-Gat1 and Pho4-Tye7) are moderately biologically plausible since they are co-annotated in the same MIPS functional categories but do not have protein-protein interactions.

\section{Performance comparison with 11 existing methods}

In this study, we adopted three performance indices to compare our predictions with 11 existing methods' predictions (Table 2). Depending on the threshold value used, different methods obtained different number of predicted cooperative TF pairs (PCTFPs). The three performance indices are introduced in following subsections, and the comparison results are also shown.

Performance index 1: The similarity of protein-protein interaction partners between the two TFs of each PCTFP

Following previous studies in the literature $[3,4,8,24]$, we evaluated cooperativity between two TFs in a PCTFP based on the rationale: the similarity of protein-protein interactions (PPI) partners between two TFs suggests that they contribute to the same biological processes and participate in the same regulatory mechanism. The physical PPI data were downloaded from the BioGRID database [25]. Given a list of PCTFPs from a method, we measured the similarity of PPI partners between the two TFs of each PCTFP by calculating a score $-\log P$, which represents the significance of their PPI partners overlap. Note that $P$ is the $\mathrm{p}$-value calculated by the hypergeometric distribution shown in the formula (1), $N_{1}$ is the number of genes whose proteins have physical PPI with the first TF, $N_{2}$ is the number the genes whose proteins have physical PPI with the second TF, $N=$ 6575 is the number of total genes in the yeast genome, and $m$ is the number of genes whose proteins have physical PPI with both TFs. The greater the $-\log P$ is, the more significant the cooperativity of a PCTFP is. To evaluate the performance of a list of PCTFPs from a method, where each PCTFP has been given a score $-\log P$, we took the mean of these scores as the final score of this performance index. Figure 2a shows that 
Table 1 The 27 predicted cooperative TF pairs

\begin{tabular}{|c|c|c|c|c|c|}
\hline Ranking & TF1 & TF2 & $\begin{array}{l}\text { Consistent with existing } \\
\text { methods' predictions }\end{array}$ & $\begin{array}{l}\text { Having } \\
\text { PPIs? }\end{array}$ & Co-annotated MIPS functional categories \\
\hline 1 & Reb1 & YDR026C & [3] & - & - \\
\hline 2 & Fkh2 & Fkh1 & {$[1],[2],[3],[4],[7],[8],[38]$} & Y & $\begin{array}{l}\text { mitotic cell cycle and cell cycle control; budding, cell polarity and filament } \\
\text { formation }\end{array}$ \\
\hline 3 & Tye7 & Cbf1 & [3] & Y & metabolism \\
\hline 4 & Dig1 & Ste12 & {$[3],[7],[20]$} & Y & $\begin{array}{l}\text { protein folding; pheromone response, mating-type determination, sex-specific } \\
\text { proteins; budding, cell polarity and filament formation }\end{array}$ \\
\hline 5 & Pdr3 & Pdr1 & - & Y & DNA binding; chemical agent resistance; detoxification \\
\hline 6 & Swi6 & Mbp1 & {$[2],[3],[4],[5],[7],[20],[38]$} & Y & $\begin{array}{l}\text { DNA synthesis and replication; mitotic cell cycle and cell cycle control; protein } \\
\text { with binding function or co-factor requirement (structural or catalytic) }\end{array}$ \\
\hline 7 & Swi6 & Swi4 & {$[2],[3],[4],[5],[7],[9],[20],[38]$} & Y & G1/S transition of mitotic cell cycle \\
\hline 8 & Dal80 & $\mathrm{G} \ln 3$ & - & Y & regulation of nitrogen metabolism \\
\hline 9 & $\mathrm{Gln} 3$ & Gat1 & [3] & Y & regulation of nitrogen metabolism \\
\hline 10 & Dal80 & Gat1 & - & - & regulation of nitrogen metabolism \\
\hline 11 & Pho4 & Cbf1 & - & Y & metabolism; DNA binding \\
\hline 12 & Pho4 & Tye7 & - & - & metabolism \\
\hline 13 & Hар3 & Hap2 & [20] & Y & regulation of C-compound and carbohydrate metabolism \\
\hline 14 & Gal80 & Gal4 & - & Y & regulation of C-compound and carbohydrate metabolism \\
\hline 15 & Met31 & Met32 & [3] & Y & $\begin{array}{l}\text { metabolism of methionine; metabolism of cysteine; regulation of amino acid } \\
\text { metabolism; regulation of nitrogen, sulfur and selenium metabolism; DNA } \\
\text { binding }\end{array}$ \\
\hline 16 & Msn4 & Msn2 & [3] & Y & DNA binding; stress response \\
\hline 17 & Rap1 & Fhl1 & {$[3],[8],[20]$} & Y & - \\
\hline 18 & Tec1 & Ste12 & [3] & Y & budding, cell polarity and filament formation \\
\hline 19 & Swi6 & Stb1 & {$[3],[7]$} & Y & G1/S transition of mitotic cell cycle \\
\hline 20 & Hap2 & Hap5 & [8] & Y & regulation of C-compound and carbohydrate metabolism \\
\hline 21 & Nrg1 & $\operatorname{Nrg} 2$ & - & Y & $\begin{array}{l}\text { protein with binding function or co-factor requirement (structural or catalytic); } \\
\text { budding, cell polarity and filament formation }\end{array}$ \\
\hline 22 & Hap3 & Hap5 & {$[8],[20]$} & Y & regulation of C-compound and carbohydrate metabolism \\
\hline 23 & Swi4 & Stb1 & {$[3],[4],[7]$} & Y & G1/S transition of mitotic cell cycle \\
\hline 24 & Swi5 & Ace2 & {$[2],[3],[4],[7],[9]$} & Y & G1 phase of mitotic cell cycle \\
\hline 25 & Sok2 & Phd1 & [3] & Y & budding, cell polarity and filament formation \\
\hline 26 & Hap5 & Hap4 & - & Y & regulation of C-compound and carbohydrate metabolism \\
\hline 27 & Mbp1 & Swi4 & {$[1],[2],[3],[6],[7],[9]$} & Y & mitotic cell cycle and cell cycle control \\
\hline
\end{tabular}

our method outperforms 10 existing methods in this score.

\section{Performance index 2: Functional similarity between the two TFs of each PCTFP}

We evaluated cooperativity between two TFs in a PCTFP based on the rationale: if two TFs have similar biological functions, then they tend to participate in the same regulatory mechanism [24]. The functional similarity score of a PCTFP is adopted from Yang et al.'s study [26], which proposed an improving GO semantic similarity measures using download random walks. The greater the functional similarity score is, the more significant the cooperativity of a PCTFP is. To evaluate the performance of a list of PCTFPs from a method, where each PCTFP has been given a functional similarity score, we took the mean of these scores as the final score of this performance index. Figure $2 \mathrm{~b}$ shows that our method outperforms 11 existing methods in this score.
Performance index 3: The significance of the overlap between $a$ list of PCTFPs from a method and a benchmark set of 27 known cooperative TF pairs

Yang et al. [9] proposed a performance index to test the prediction accuracy of different methods by comparing the significance of the overlap of different lists of PCTFPs with a benchmark set of known cooperative TF pairs. The benchmark set (Table 3) has 27 TF pairs, which is complied from the MIPS transcription complex catalog [27]. As far as we know, this is the only highquality dataset of TF cooperativity currently available [9]. Then given a list of predicted cooperative TF pairs from a method, we calculated the significance of the overlap of this list with the benchmark set using Yang et al.'s index. For the given list, a score which represents the significance of the overlap is defined as the $-\log P$, where $P$ is the $p$-value computed using Fisher exact test. The higher the score, the better the performance. 
Table 2 The 11 compared existing methods.

\begin{tabular}{|c|c|c|c|c|}
\hline $\begin{array}{l}\text { Existing } \\
\text { methods }\end{array}$ & Data sources used & Method description & $\begin{array}{l}\text { Threshold } \\
\text { of p-value }\end{array}$ & $\begin{array}{l}\text { Number of } \\
\text { predicted } \\
\text { cooperative TF } \\
\text { pairs }\end{array}$ \\
\hline $\begin{array}{l}\text { Wang et al. } \\
(2006)[38]\end{array}$ & $\begin{array}{l}\text { ChIP-chip data, gene } \\
\text { expression data, TFBS } \\
\text { data }\end{array}$ & $\begin{array}{l}\text { They developed a new framework to infer the combinatorial control of } \\
\text { TFs by integrating heterogeneous functional genomic datasets. }\end{array}$ & $10^{-3}$ & 14 \\
\hline $\begin{array}{l}\text { Tsai et al. } \\
\text { (2005) [5] }\end{array}$ & $\begin{array}{l}\text { ChIP-chip data, gene } \\
\text { expression data }\end{array}$ & $\begin{array}{l}\text { They used statistical methods to identify yeast cell cycle TFs and } \\
\text { synergistic pairs of TFs. }\end{array}$ & $10^{-5}$ & 18 \\
\hline $\begin{array}{l}\text { Elati et al. } \\
\text { (2007) [28] }\end{array}$ & Gene expression data & $\begin{array}{l}\text { They adopted a data mining system to learn transcriptional regulation } \\
\text { relationship from gene expression data. }\end{array}$ & $10^{-3}$ & 20 \\
\hline $\begin{array}{l}\text { Nagamine et } \\
\text { al. (2005) [8] }\end{array}$ & ChIP-chipdata, PPI data & $\begin{array}{l}\text { They inferred the cooperative pairs under the assumption that the } \\
\text { existence of interaction between two proteins suggests that they } \\
\text { contribute to the same or similar biological process. }\end{array}$ & $10^{-3}$ & 24 \\
\hline $\begin{array}{l}\text { Datta and } \\
\text { Zhao (2007) } \\
{[2]}\end{array}$ & ChIP-chip data & $\begin{array}{l}\text { They used a log-linear model to study cooperative binding among TFs } \\
\text { and developed an Expectation-Maximization algorithm for statistical } \\
\text { inferences. }\end{array}$ & $10^{-3}$ & 25 \\
\hline $\begin{array}{l}\text { He et al. } \\
\text { (2006) [6] }\end{array}$ & $\begin{array}{l}\text { ChIP-chip data, gene } \\
\text { expression data }\end{array}$ & $\begin{array}{l}\text { They adopted the microarray expression data to predict the } \\
\text { cooperative TF pairs by testing whether the expression of target genes } \\
\text { was significantly influenced by their cooperative effect with the } \\
\text { multivariate method, ANOVA. }\end{array}$ & $10^{-2}$ & 30 \\
\hline $\begin{array}{l}\text { Banerjee and } \\
\text { Zhang (2003) } \\
{[4]}\end{array}$ & $\begin{array}{l}\text { ChIP-chip data, gene } \\
\text { expression data }\end{array}$ & $\begin{array}{l}\text { They infer the cooperative pairs under the assumption that a pair of } \\
\text { TFs is cooperative if genes regulated by both TFs are more co- } \\
\text { expressed than those genes regulated by either TF alone. }\end{array}$ & $5 \times 10^{-2}$ & 31 \\
\hline $\begin{array}{l}\text { Chang et al. } \\
\text { (2006) [7] }\end{array}$ & $\begin{array}{l}\text { ChIP-chip data, gene } \\
\text { expression data }\end{array}$ & They employed a stochastic system model to assess TF cooperativity. & $10^{-21}$ & 55 \\
\hline $\begin{array}{l}\text { Yang et al. } \\
\text { (2010) [9] }\end{array}$ & $\begin{array}{l}\text { ChIP-chip data, TF } \\
\text { knockout data }\end{array}$ & $\begin{array}{l}\text { They predicted cooperativity between TFs by identifying the most } \\
\text { statistically significant overlap of target genes regulated by two TFs in } \\
\text { ChIP-chip data and TF knockout data. }\end{array}$ & $5 \times 10^{-3}$ & 186 \\
\hline $\begin{array}{l}\text { Chen et al. } \\
\text { (2012) [3] }\end{array}$ & ChIP-chip data & $\begin{array}{l}\text { They facilitated identification of interactions between TFs by using } \\
\text { motif discovery method when detecting overlapping targets of TFs } \\
\text { based on ChIP-chip data. }\end{array}$ & $10^{-3}$ & 221 \\
\hline $\begin{array}{l}\text { Yu et al. } \\
\text { (2006) [1] }\end{array}$ & ChIP-chip data & $\begin{array}{l}\text { They proposed a method: Motif-PIE, which predicts interacting TF pairs } \\
\text { by using a motif discovery procedure. }\end{array}$ & $10^{-8}$ & 300 \\
\hline
\end{tabular}

The table shows the information of 11 existing methods adopted for comparison. The data sources used, a brief description of the method, the p-value threshold and the number of predicted cooperative TF pairs of each method are described in columns 2, 3, 4 and 5.

Figure 2c shows that our method outperforms 11 existing methods in this score.

\section{Discussion}

\section{Our method is robust against different thresholds of the} cooperativity score

In this study, we set a threshold of cooperativity score to be 120 and reported 27 PCTFPs whose cooperativity scores are larger than the threshold. The number of PCTFPs reported by our method is similar to those of five previous methods $[2,4,6,8,28]$. To check the robustness of our method against different thresholds of the cooperativity score, we tested four different thresholds $(125,120,110$ and 90). Figure 3 shows that no matter which threshold is used, the performance of our method is always the same (i.e. superior to 10 out of 11 existing methods) on the performance index 1 . This suggests that our method is robust against different thresholds of the cooperativity score.
Our method is robust against different qualities of TFBS data The quality of TFBS data retrieved from SwissRegulon database depends on the posterior probability threshold being used. In this study, a threshold 0.3 of the posterior probability was applied to select putative TFBSs. To check the robustness of our method against different qualities of TFBS data, we tested four different posterior probability thresholds $(0.2,0.3,0.4$ and 0.5$)$. Figure 4 shows that no matter which threshold is used, the performance of our method is always the same (i.e. superior to 10 out of 11 existing methods) on the performance index 1 . This suggests that our method is robust against different thresholds of posterior probability to control the quality of TFBS data.

\section{Our method outperforms existing methods in the precision and recall when using a benchmark set of 27 known cooperative TF pairs}

3In the performance index 3 , the significance of the overlap of the list of PCTFPs from a method with the 


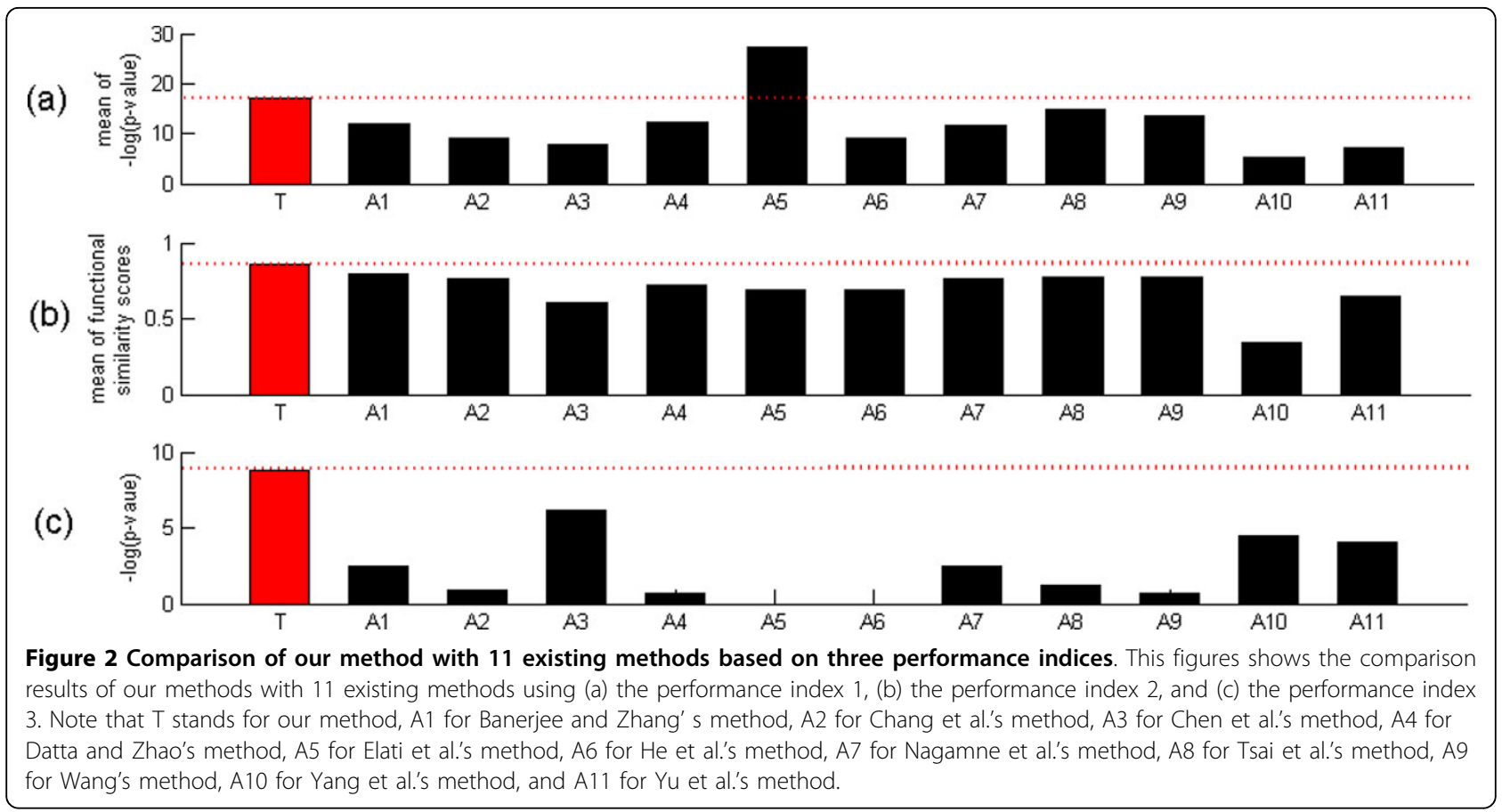

benchmark set of 27 known cooperative TF pairs is used to evaluate the performance of a method. Here, we use the precision and recall to evaluate the performance of a method. As shown in Figure 5, our method outperforms 11 existing methods in the precision and outperforms 10 existing methods in the recall.

The nucleosome occupancy data contributes to the overall improved prediction

To demonstrate that the nucleosome occupancy data contributes to the overall improved prediction, we tested our method when nucleosome data are used (denoted as $\mathrm{T} \mathrm{w} / \mathrm{Nucleosome}$ ) and when nucleosome data are not used (denoted as $\mathrm{T}$ w/o Nucleosome) on the performance index 1. As shown in Figure 6, the $-\log P$ of $\mathrm{T}$ w/ Nucleosome is higher than that of $\mathrm{T}$ w/o Nucleosome by 1.23 , meaning that the $\mathrm{p}$-value of $\mathrm{T} \mathrm{w} /$ Nucleosome is less than that of $\mathrm{T}$ w/o Nucleosome by more than 10 folds. This suggests that nucleosome occupancy data do contribute to the overall improved prediction of our method.

Issue of applying our method to other model organisms Our method is used to infer the cooperativity between two yeast TFs by integrating the TF-gene documented regulation, TFBS and nucleosome occupancy data. Theoretically, it can be applied to other model organisms which also have these three kinds of genome-wide data. There are two reasons why we used yeast as the model organism to test our method. First, yeast is the only organism which has more than 206000 TF-gene documented regulation data available. The useful TF-gene documented regulation data [29], which provide the experimentally validated regulatory relationships between TFs and genes, are collected from more than 1300 published papers by the team of the YEASTRACT database [18]. Second, yeast is the only organism that is tested by more than 10 existing algorithms. Therefore, using yeast as the model organism makes it possible to compare our predictions with the predictions of many existing methods.

\section{A cooperative TF Network}

Figure 7 shows a cooperative TF network constructed from our 27 predicted cooperative TF pairs. This cooperative TF network has four main groups which belong to four biological processes according to MIPS functional categories. More precisely, there are (i) 12 pairs annotated in metabolism, (ii) 7 pairs annotated in cell cycle, (iii) 5 pairs annotated in cell type differentiation, and (iv) 2 pairs annotated in cell rescue, defense and virulence. This demonstrates that our method has the power to find cooperative TF pairs of different biological processes.

\section{Cell cycle}

Since cell cycle process has been well investigated in the literature, let us discuss it in more details. Cell cycle is a complex process and it consists of four main phases: G1, S, G2 and $M$. 
Table 3 The benchmark set of 27 known cooperative TF pairs.

\begin{tabular}{|c|c|c|}
\hline TF pairs & MIPS complex ID & MIPS complex name \\
\hline ARG80-MCM1 & 510.190 .120 & ARG complex \\
\hline MET4-MET28 & $\begin{array}{l}510.190 .160 .10 \\
510.190 .160 .20 \\
510.190 .160 .30\end{array}$ & $\begin{array}{l}\text { Cbf1/Met4/Met28 complex } \\
\text { Met4/Met28/Met31 complex } \\
\text { Met4/Met28/Met32 complex }\end{array}$ \\
\hline STP4-STP1 & 440.30 .30 & tRNA splicing \\
\hline IME1-UME6 & 510.190 .200 & Ume6/Ime1 complex \\
\hline HAP5-HAP4 & 510.160 & CCAAT-binding factor complex \\
\hline STP2-STP1 & 440.30 .30 & tRNA splicing \\
\hline HAP2-HAP3 & 510.160 & CCAAT-binding factor complex \\
\hline ARG80-ARG81 & 510.190 .120 & ARG complex \\
\hline MET4-MET31 & 510.190 .160 .20 & Met4/Met28/Met31 complex \\
\hline CBF1-MET28 & 510.190 .160 .10 & Cbf1/Met4/Met28 complex \\
\hline MCM1-ARG81 & 510.190 .120 & ARG complex \\
\hline HAP5-HAP2 & 510.160 & CCAAT-binding factor complex \\
\hline HAP4-HAP2 & 510.160 & CCAAT-binding factor complex \\
\hline PIP2-OAF1 & 510.190 .100 & OAF complex \\
\hline MET4-CBF1 & 510.190 .160 .10 & Cbf1/Met4/Met28 complex \\
\hline GCR1-GCR2 & 510.190 .90 & GCR complex \\
\hline RTG1-RTG3 & 510.190 .130 & RTG complex \\
\hline SWI6-MBP1 & 510.190 .70 & MBF complex \\
\hline HAP5-HAP3 & 510.160 & CCAAT-binding factor complex \\
\hline HAP4-HAP3 & 510.160 & CCAAT-binding factor complex \\
\hline GAL3-GAL80 & 510.190 .80 & GAL80 complex \\
\hline MET4-MET32 & 510.190 .160 .30 & Met4/Met28/Met32 complex \\
\hline STP4-STP2 & 440.30 .30 & tRNA splicing \\
\hline SWI4-SWI6 & 510.190 .60 & SBF complex \\
\hline GAL80-GAL4 & 510.190 .80 & GAL80 complex \\
\hline MET32-MET28 & 510.190 .160 .30 & Met4/Met28/Met32 complex \\
\hline MET28-MET31 & 510.190 .160 .20 & Met4/Met28/Met31 complex \\
\hline
\end{tabular}

The list of 27 known cooperative TF pairs are derived from biochemically well-defined transcriptional complexes within the MIPS complex catalogue.

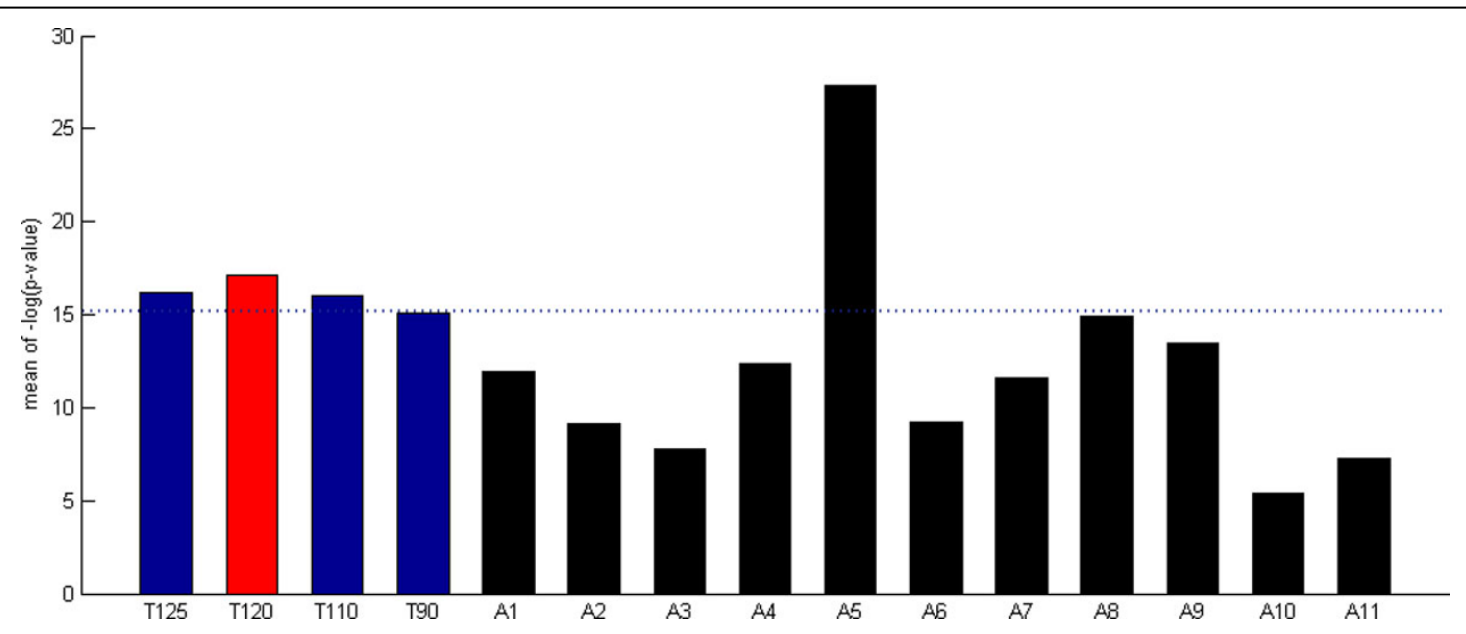

Figure 3 The performance of our method when using different thresholds of the cooperativity score. To check the robustness of our method against different thresholds of cooperativity scores, we tested four different thresholds $(125,120,110$ and 90). The figure shows that no matter which threshold is used, the performance of our method is always the same (i.e. superior to 10 out of 11 existing methods) on the performance index 1 . This suggests that our method is robust against different thresholds of the cooperativity score. 

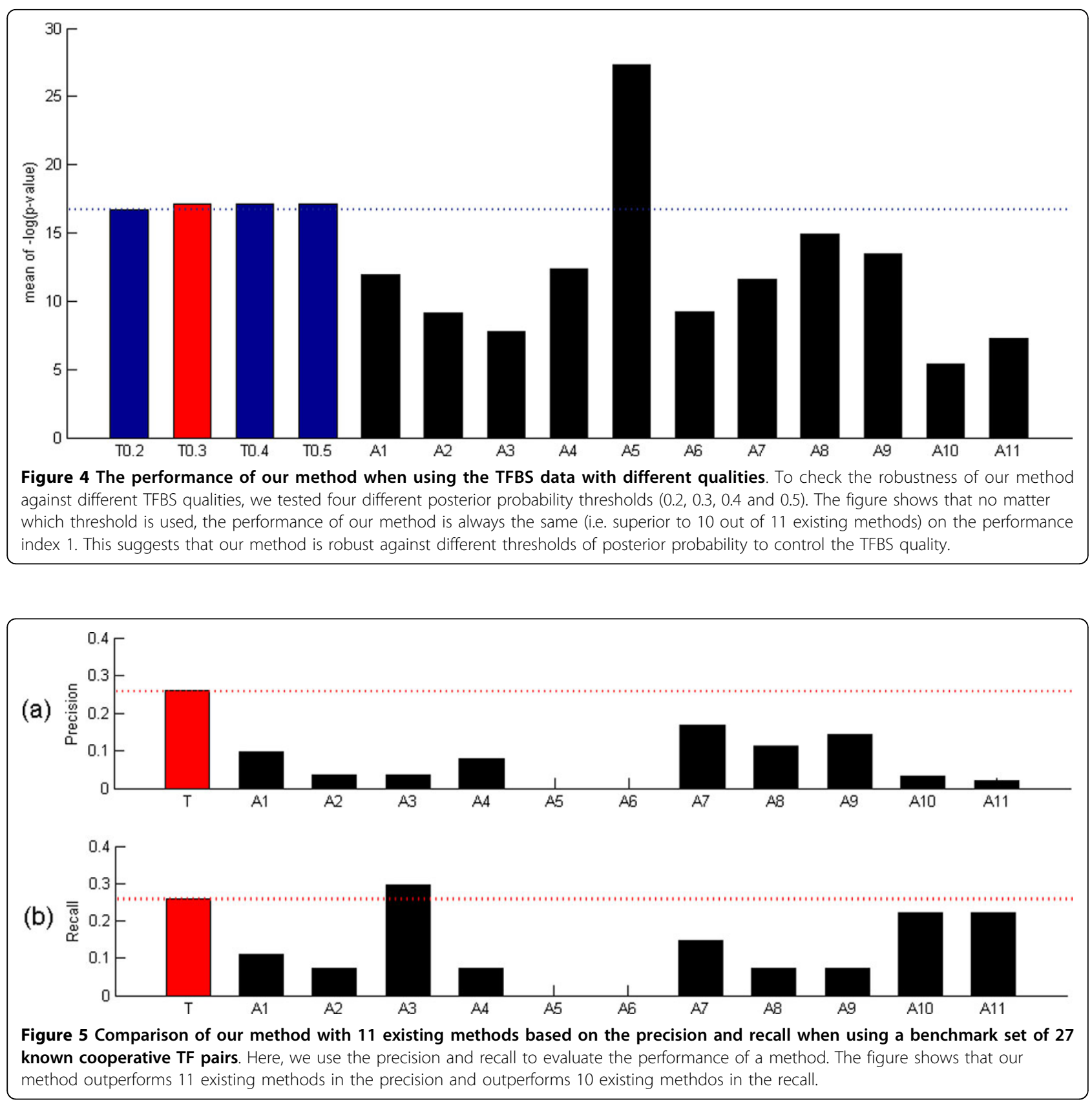

\section{G1/S phase}

The G1 to S phase transition of the eukaryotic cell cycle is crucial to the coordination of cell cycle progression with cellular growth. During the G1/S transition of the cell cycle in yeast, SBF and MBF are known to activate gene expression [30]. SBF is a protein complex composed of Swi4 and Swi6, and MBF is a protein complex composed of Mbp1 and Swi6 [31]. Our method successfully predicted the cooperativity between Swi4 and Swi6, and the cooperativity between Mbp1 and Swi6 (see
Figure 7). Moreover, Mbp1 is known to related to Swi4 because the MBP1 SWI4 double knockout strain were inviable. The cooperativity between Mbp1 and Swi4 is successfully captured by our method (see Figure 7). In addition, a study suggests that Stb1 may affect MBFdependent transcription [32]. Stb1 is a protein which regulates the timing of start transcription in the absence of the G1 regulator Cln3. The cooperativity between Stb1 and Swi6 (a member of MBF) is successfully identified by our method (see Figure 7). 

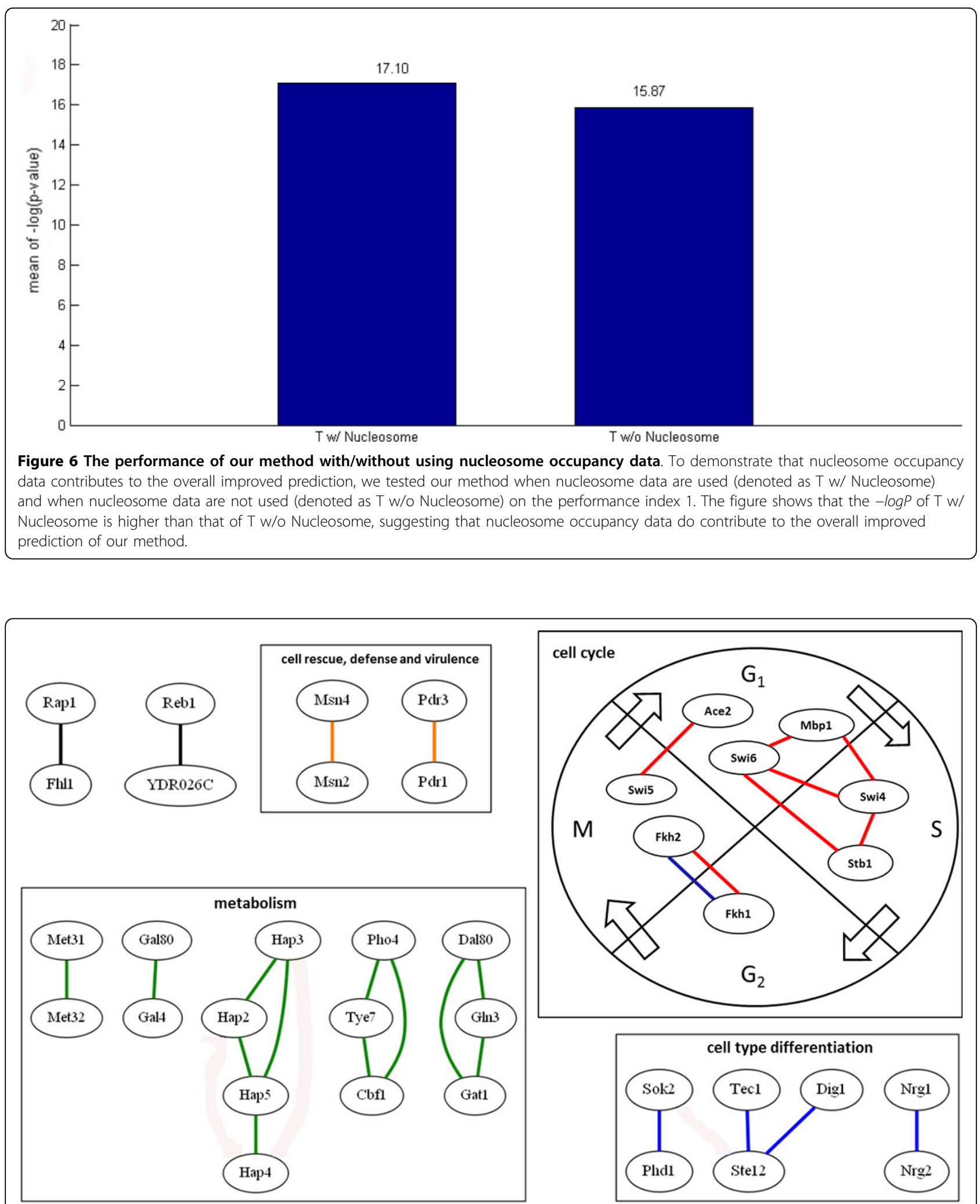

Figure 7 A TF cooperativity network. A TF cooperativity network is constructed from our 27 predicted cooperative TF pairs. Nodes represent TFs. A black line between two TFs means that these two TFs are predicted to be a cooperative TF pair but they are not annotated in the same MIPS functional category. A colored line between two TFs means that these two TFs are predicted to be a cooperative TF pair and they are annotated in the same MIPS functional category. The green color stands for metabolism, red for cell cycle, blue for cell type differentiation, orange for cell rescue, defense and virulence. 


\section{G2/M phase}

In the G2/M phase, Fkh1 and Fkh2 play essential roles in the activation of the CLB2 cluster genes and they share $72 \%$ identical DNA binding domain. Moreover, the double mutant of FKH1 and FKH2 displays obvious morphological change [33-36]. Our method successfully predicts the cooperativity between Fkh1 and Fkh2 (see Figure 7).

\section{M/G1 phase}

In M/G1 phase, Ace2 and Swi5 co-regulate the expression of many cell cycle genes in yeast [37]. Moreover, Ace 2 and Swi5 proteins show similarity at the amino acid level and bind to the same DNA sequence with $82 \%$ identical DNA binding domains. Our method successfully identifies the cooperativity between Ace2 and Swi5 (see Figure 7).

\section{Conclusions}

In this paper, we developed a method to infer the cooperativity between two TFs by integrating the TF-gene documented regulation, TFBS and nucleosome occupancy data. Two TFs are predicted as cooperative if (i) they have a significantly higher number of common target genes than random expectation and (ii) their binding sites (in the promoters of their common target genes) tend to be codepleted of nucleosomes in order to make these binding sites simultaneously accessible to TF binding. A list of 27 cooperative TF pairs has been predicted by our method. Among these 27 predicted cooperative TF pairs, 19 pairs are also predicted by existing methods. The other 8 pairs are novel cooperative TF pairs. The biological relevance of these 8 novel cooperative TF pairs is justified by the existence of protein-protein interactions and co-annotation in the same MIPS functional categories. Moreover, our method is shown to outperform the 11 existing methods based on the three performance indices: (i) the similarity of protein-protein interaction partners between two TFs, (ii) the functional similarity between two TFs, and (iii) the overlap between a method's prediction result and the benchmark set of 27 known cooperative TF pairs. Finally, the cooperative TF network constructed from the 27 predicted pairs demonstrates that our method has the power to find cooperative TF pairs of different biological processes. We believe that our prediction will help biologists to understand the mechanism of transcriptional regulation in eukaryotic cells.

\section{Competing interests}

The authors declare that they have no competing interests.

\section{Authors' contributions}

WSW conceived the research topic and provided essential guidance. WSW FJL and $\mathrm{MHJ}$ developed the algorithm and wrote the manuscript. FJL, MHJ and CCC performed all the simulations. YMH provided advices on the manuscript writing. All authors have read and approved the final manuscript.

\section{Acknowledgements}

This study was supported by National Cheng Kung University and Ministry of Science and Technology of Taiwan MOST-103-2221-E-006-174-MY2. This article has been published as part of BMC systems Biology Volume 8 Supplement 5, 2014: Proceedings of the 25th International Conference on Genome Informatics (GIW/ISCB-Asia): Systems Biology. The full contents of the supplement are available online at http://www.biomedcentral.com/ bmcsystbiol/supplements/8/S5.

\section{Declarations}

The publication charges of this article were funded by Ministry of Science and Technology of Taiwan MOST-103-2221-E-006-174-MY2.

\section{Authors' details}

'Department of Engineering Science, National Cheng Kung University, No.1 University Road, 70101, Tainan, Taiwan. ${ }^{2}$ Department of Electrical Engineering, National Cheng Kung University, No.1 University Road, 70101, Tainan, Taiwan.

Published: 12 December 2014

\section{References}

1. Yu X, Lin J, Masuda T, Esumi N, Zack DJ, Qian J: Genome-wide prediction and characterization of interactions between transcription factors in Saccharomyces cerevisiae. Nucleic Acids Res 2006, 34(3):917-927.

2. Datta $\mathrm{D}$, Zhao $\mathrm{H}$ : Statistical methods to infer cooperative binding among transcription factors in Saccharomyces cerevisiae. Bioinformatics 2008, 24(4):545-552.

3. Chen MJM, Chou LC, Hsieh TT, Lee DD, Liu KW, Yu CY, Oyang YJ, Tsai HK, Chen CY: De novo motif discovery facilitates identification of interactions between transcription factors in Saccharomyces cerevisiae. Bioinformatics 2012, 28(5):701-708.

4. Banerjee $\mathrm{N}$, Zhang MQ: Identifying cooperativity among transcription factors controlling the cell cycle in yeast. Nucleic Acids Res 2003, 31(23):7024-7031.

5. Tsai HK, Lu HHS, Li WH: Statistical methods for identifying yeast cell cycle transcription factors. Proc Natl Acad Sci USA 2005, 102(38):13532-13537.

6. He D, Zhou D, Zhou Y: Identifying synergistic transcriptional factors involved in the yeast cell cycle using microarray and ChIP-chip data. Proceedings of the Fifth International Conference on Grid and Cooperative Computing Workshops GCCW '06, Washington, DC, USA: IEEE Computer Society; 2006, 357-360.

7. Chang $\mathrm{YH}$, Wang YC, Chen BS: Identification of transcription factor cooperativity via stochastic system model. Bioinformatics 2006, 22(18):2276-2282.

8. Nagamine N, Kawada Y, Sakakibara Y: Identifying cooperative transcriptional regulations using protein-protein interactions. Nucleic Acids Res 2005, 33(15):4828-4837.

9. Yang $Y$, Zhang Z, Li Y, Zhu XG, Liu Q: Identifying cooperative transcription factors by combining ChIP-chip data and knockout data. Cell Res 2010, 20(11):1276-1278.

10. Pilpel $Y$, Sudarsanam $P$, Church GM: Identifying regulatory networks by combinatorial analysis of promoter elements. Nat Genet 2001, 29(2):153-159.

11. Wang Y, Zhang XS, Xia Y: Predicting eukaryotic transcriptional cooperativity by Bayesian network integration of genome-wide data. Nucleic Acids Res 2009, 37(18):5943-5958.

12. Hu P, Shen $Z$, Tu H, Zhang L, Shi T: Integrating multiple resources to identify specific transcriptional cooperativity with a Bayesian approach. Bioinformatics 2014, 30(6):823-830.

13. Daenen F, Van Roy F, De Bleser PJ: Low nucleosome occupancy is encoded around functional human transcription factor binding sites. BMC Genomics 2008, 9:332.

14. Lin Z, Wu WS, Liang H, Woo Y, Li WH: The spatial distribution of cis regulatory elements in yeast promoters and its implications for transcriptional regulation. BMC Genomics 2010, 11:581.

15. Yi X, Cai YD, He Z, Cui W, Kong X: Prediction of nucleosome positioning based on transcription factor binding sites. PLOS One 2010, 5(9):e12495.

16. Swamy K, Chu WY, Wang CY, Tsai HK, Wang D: Evidence of association between nucleosome occupancy and the evolution of transcription factor binding sites in yeast. BMC Evol Biol 2011, 11:150. 
17. Mavrich TN, loshikhes IP, Venters BJ, Jiang C, Tomsho LP, Qi J, Schuster SC, Albert I, Pugh BF: A barrier nucleosome model for statistical positioning of nucleosomes throughout the yeast genome. Genome Res 2008, 18(7):1073-1083.

18. Abdulrehman D, Monteiro PT, Teixeira MC, Mira NP, Louren, $c 0$ AB, dos Santos SC, Cabrito TR, Francisco AP, Madeira SC, Aires RS, Oliveira AL, Sá-Correia I, Freitas AT: YEASTRACT: providing a programmatic access to curated transcriptional regulatory associations in Saccharomyces cerevisiae through a web services interface. Nucleic Acids Res 2011, 39(suppl 1):D136-D140.

19. Pachkov M, Erb I, Molina N, Van Nimwegen E: SwissRegulon: a database of genome-wide annotations of regulatory sites. Nucleic Acids Res 2007, 35(suppl 1):D127-D131.

20. Garten Y, Kaplan S, Pilpel Y: Extraction of transcription regulatory signals from genome-wide DNA-protein interaction data. Nucleic Acids Res 2005, 33(2):605-615.

21. Bernstein BE, Liu CL, Humphrey EL, Perlstein EO, Schreiber SL: Global nucleosome occupancy in yeast. Genome Biol 2004, 5(9):R62.

22. Wu WS, Li WH: Systematic identification of yeast cell cycle transcription factors using multiple data sources. BMC Bioinformatics 2008, 9:522.

23. Yang TH, Wu WS: Identifying biologically interpretable transcription factor knockout targets by jointly analyzing the transcription factor knockout microarray and the ChIP-chip data. BMC Syst Biol 2012, 6:102.

24. Lai F, Chang H, Huang Y, Wu W: A comprehensive performance evaluation on the prediction results of existing cooperative transcription factors identification algorithms. BMC Syst Biol 2014, 8(S4):S9.

25. Stark C, Breitkreutz BJ, Reguly T, Boucher L, Breitkreutz A, Tyers M: BioGRID: a general repository for interaction datasets. Nucleic Acids Res 2006, 34(suppl 1):D535-D539.

26. Yang H, Nepusz T, Paccanaro A: Improving GO semantic similarity measures by exploring the ontology beneath the terms and modelling uncertainty. Bioinformatics 2012, 28(10):1383-1389.

27. Mewes HW, Frishman D, Güldener U, Mannhaupt G, Mayer K, Mokrejs M, Morgenstern B, Münsterkötter M, Rudd S, Weil B: MIPS: a database for genomes and protein sequences. Nucleic Acids Res 2002, 30:31-34.

28. Elati $M$, Neuvial P, Bolotin-Fukuhara M, Barillot E, Radvanyi F, Rouveirol C: LICORN: learning cooperative regulation networks from gene expression data. Bioinformatics 2007, 23(18):2407-2414.

29. Yang TH, Wang CC, Wang YC, Wu WS: YTRP: a repository for yeast transcriptional regulatory pathways. Database (Oxford) 2014.

30. Iyer VR, Horak CE, Scafe CS, Botstein D, Snyder M, Brown PO: Genomic binding sites of the yeast cell-cycle transcription factors SBF and MBF. Nature 2001, 409(6819):533-538.

31. Koch C, Moll T, Neuberg M, Ahorn H, Nasmyth K: A role for the transcription factors Mbp1 and Swi4 in progression from G1 to $S$ phase. Science 1993, 261(5128):1551-1557.

32. Costanzo M, Schub O, Andrews B: G1 transcription factors are differentially regulated in Saccharomyces cerevisiae by the Swi6-binding protein Stb1. Mol Cell Biol 2003, 23(14):5064-5077.

33. Koranda M, Schleiffer A, Endler L, Ammerer G: Forkhead-like transcription factors recruit Ndd1 to the chromatin of G2/M-specific promoters. Nature 2000, 406(6791):94-98

34. Kumar R, Reynolds DM, Shevchenko A, Shevchenko A, Goldstone SD, Dalton S: Forkhead transcription factors, Fkh1p and Fkh2p, collaborate with Mcm1p to control transcription required for M-phase. Curr Biol 2000, 10(15):896-906

35. Pic A, Lim FL, Ross SJ, Veal EA, Johnson AL, Sultan MR, West AG, Johnston LH, Sharrocks AD, Morgan BA: The forkhead protein Fkh2 is a component of the yeast cell cycle transcription factor SFF. EMBO J 2000, 19(14):3750-3761.

36. Futcher B: Transcriptional regulatory networks and the yeast cell cycle. Curr Opin Cell Biol 2002, 14(6):676-683.

37. Doolin MT, Johnson AL, Johnston LH, Butler G: Overlapping and distinct roles of the duplicated yeast transcription factors Ace2p and Swi5p. Mol Microbiol 2001, 40(2):422-432

38. Wang J: A new framework for identifying combinatorial regulation of transcription factors: a case study of the yeast cell cycle. J Biomed Inform 2007, 40(6):707-725.
doi:10.1186/1752-0509-8-S5-S2

Cite this article as: Lai et al:: Identifying cooperative transcription

factors in yeast using multiple data sources. BMC Systems Biology 20148

(Suppl 5):S2.

\section{Submit your next manuscript to BioMed Central and take full advantage of:}

- Convenient online submission

- Thorough peer review

- No space constraints or color figure charges

- Immediate publication on acceptance

- Inclusion in PubMed, CAS, Scopus and Google Scholar

- Research which is freely available for redistribution

Submit your manuscript at www.biomedcentral.com/submit
Ciomed Central 